\title{
Placement of self-expandable metallic stents for tracheal stenosis secondary to thyroid cancer
}

\author{
RENA YUASA $^{1}$, YOSHINOBU HATA ${ }^{1}$, HAJIME OTSUKA ${ }^{1}$, TAKASHI MAKINO ${ }^{1}$, SATOSHI KOEZUKA ${ }^{1}$, \\ FUMITOMO SATO $^{1}$, KAZUYOSHI TAMAKI ${ }^{1}$, SHUICHI SASAMOTO ${ }^{1}$, KEIGO TAKAGI $^{2}$ and AKIRA IYODA ${ }^{1}$ \\ ${ }^{1}$ Division of Chest Surgery, Toho University School of Medicine, Tokyo 143-8541; \\ ${ }^{2}$ Nishi Yokohama International Hospital, Yokohama, Kanagawa 245-0062, Japan
}

Received May 23, 2014; Accepted July 3, 2014

DOI: $10.3892 / \mathrm{mco} .2014 .350$

\begin{abstract}
The indications and suitable approaches for treating upper airway obstruction secondary to thyroid cancer are controversial. Patients with thyroid cancer generally have a good prognosis, but airway stenosis and vocal cord paralysis are not uncommon. Subglottic airway stenting may be challenging, due to stent migration, granulation tissue formation and supraglottic stenosis. In this study, we evaluated the application of covered self-expandable metallic stents to relieve upper airway obstruction. This was a retrospective study of 5 patients with airway stenosis due to thyroid cancer treated in 2009 and 2010. Immediate airway enlargement was achieved in 3 patients with stenosis at the middle mediastinum. Gradual enlargement over 2 months was observed in the remaining 2 patients with stenosis at the cervical level. The performance status was improved in all 5 patients, including a case with anaplastic carcinoma. The follow-up averaged 13 months (range, 8-27 months). Granulation tissue developed at both ends of the stent in 3 patients, sputum was retained in 2 cases and bacterial colonization was detected in all 5 cases. No stent migration was reported. Additional tracheostomy was required in 2 patients, due to proximal tumor growth or progressive bilateral vocal cord paralysis after 10 and 6 months, respectively. In conclusion, stenting for central airway stenosis secondary to thyroid cancer may be beneficial, even in patients with anaplastic carcinoma. Long-term regular bronchoscopic
\end{abstract}

Correspondence to: Dr Yoshinobu Hata, Division of Chest Surgery, Toho University School of Medicine, 6-11-1 Omori-nishi, Tokyo 143-8541, Japan

E-mail: hata@med.toho-u.ac.jp

Abbreviations: CT, computed tomography; 3D, three-dimensional; PS, performance status; $\mathrm{PEF}$, peak expiratory flow; $\% \mathrm{FEV}_{1.0}$, percent predicted forced expiratory volume in $1.0 \mathrm{sec}$; PSL, prednisolone; MMC, mitomycin C; P. aeruginosa, Pseudomonas aeruginosa; MRSA, methicillin-resistant Staphylococcus aureus; K. oxytoca, Klebsiella oxytoca; St. pneumoniae, Streptococcus pneumoniae

Key words: self-expandable metallic stents, tracheal stenosis, airway obstruction, thyroid cancer, flexible bronchoscopy follow-up is required to monitor complications, as patients with thyroid cancer are at high risk of granulation tissue formation, sputum retention and bacterial colonization.

\section{Introduction}

In Japan 8,069 individuals (2,023 men and 6,046 women) were diagnosed with thyroid cancer in 2003, of whom 1,427 (447 men and 980 women) succumbed to their disease. Thus, the crude death rate is estimated to be 0.84 and 1.61 per 100,000 in men and women, respectively (1). Over $90 \%$ of the patients have histologically well-differentiated cancer, with a good prognosis regarding long-term survival. Noguchi et al (2) reported that $>74.4 \%$ of male and $>88.8 \%$ of female patients with thyroid papillary cancer in the poor risk group survived for $>10$ years. However, the terminal stage of the disease may be associated with airway stenosis secondary to thyroid enlargement, which, according to the English-language literature, may occur in $16-60 \%$ of the patients (3).

Upper airway stenosis secondary to thyroid cancer exhibits characteristics that distinguish it from that caused by other malignant tumors. First, the slowly progressing nature of well-differentiated thyroid cancer tends to mask the symptoms and results in acute onset of severe airway obstruction. Second, this type of tracheal stenosis is often associated with tracheal compression caused by the solid thyroid cancer tissue surrounding the trachea in the limited space of the thoracic inlet; this means that sufficient enlargement of the airway cannot be achieved immediately after stent placement, but time is required for the stent to expand and open up the airway. Third, thyroid cancer invasion may cause vocal cord paralysis, resulting in difficulties with sputum expectoration and a deteriorating state of dyspnea. Even when airway enlargement is achieved after stent placement, dyspnea may not sufficiently improve in the presence of vocal cord paralysis, particularly when this is bilateral.

Although severe obstruction of the airway secondary to thyroid cancer may be considered as a symptom of terminal-stage disease, it may be quite extensive. Airway stenting may relieve the symptoms of respiratory impairment and play an important role in palliative medicine. In this study, we aimed to evaluate the efficacy of self-expandable metallic stents for relieving central airway stenosis secondary to thyroid 


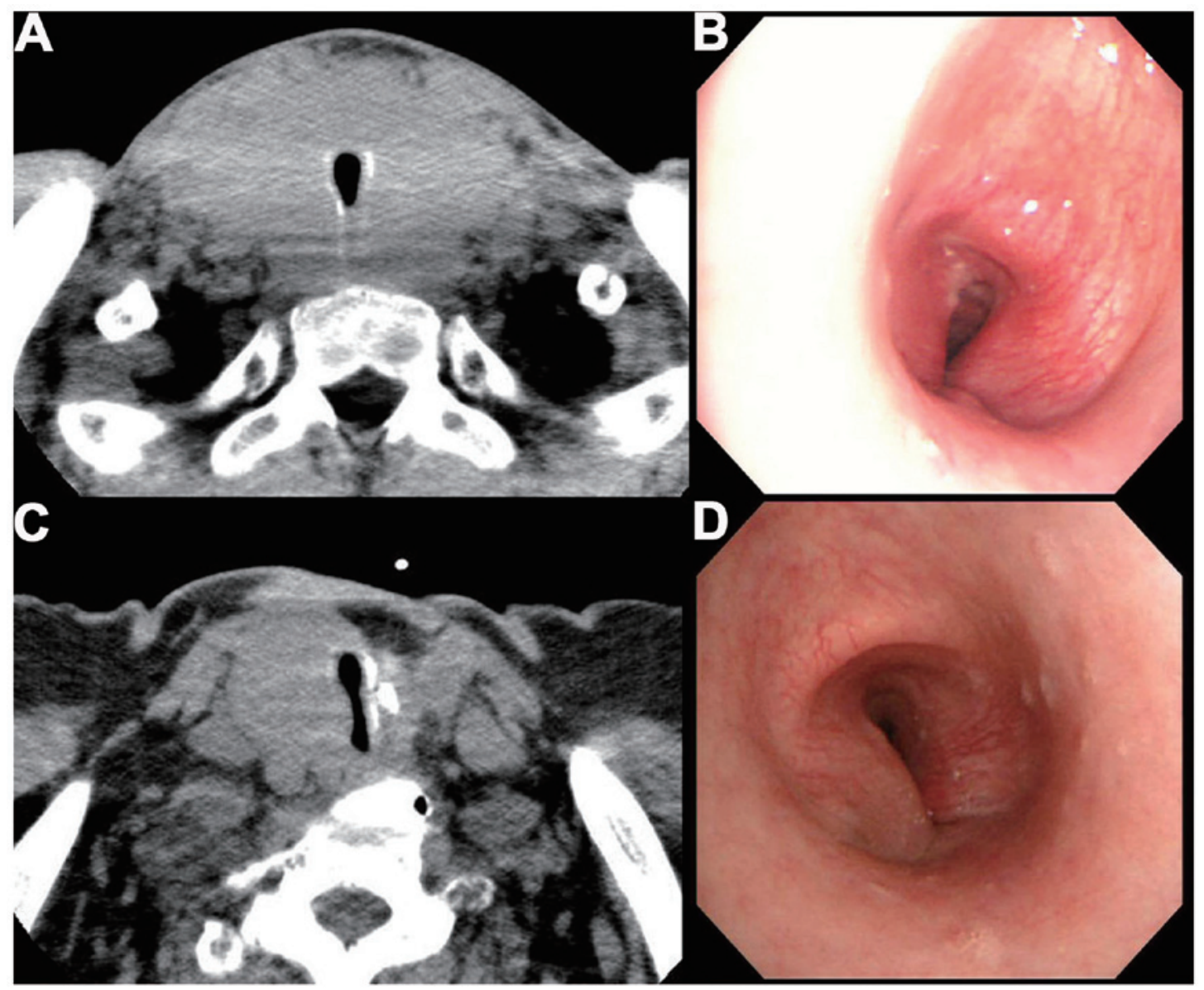

Figure 1. Computed tomography and bronchoscopic images of case 1 (A and B) and case 2 (C and D).

cancer and the complications associated with long-term stent placement.

\section{Patients and methods}

Patients. A retrospective study was conducted of 5 patients with thyroid cancer, who presented with central airway stenosis caused by thyroid cancer invasion or lymph node metastases. The characteristics of the patients, who were recruited in 2009 and 2010, are summarized in Table I. The indications for interventional bronchoscopy were acute stridor leading to respiratory insufficiency and surgical inoperability. The 5 patients were divided into two groups, according to the location of the stenosis (Table II). Group A had tracheal stenosis at the cervical level, caused by thyroid cancer (Fig. 1) and group B had stenosis at the mediastinum, caused by thyroid cancer or metastatic lymph nodes. The minimal diameter of the central airway was $2-3 \mathrm{~mm}$ and the length of the stenosis was $2-7 \mathrm{~cm}$ (Table II). Four of the 5 patients also exhibited vocal cord paralysis.

Approval for this study was granted by the Institutional Review Board of Toho University Omori Medical Center (assurance no. 25-39).

Patient workup. Prior to stenting, computed tomography (CT), three-dimensional (3D) CT and bronchoscopy were performed to evaluate the diameter, length and location of the stenosis. Spirometry with peak-flow analysis and interviewing were performed to evaluate the objective and subjective symptoms, performance status (PS), peak expiratory flow (PEF) and percent predicted forced expiratory volume in $1.0 \mathrm{sec}\left(\% \mathrm{FEV}_{1.0}\right)$. After stenting, the same procedures were performed periodically to assess the condition of the stents and monitor complications. Written informed consent for airway stenting was obtained from each patient.

Stenting procedures. All the procedures were performed under general anesthesia in an operating theater equipped with an X-ray fluoroscope and with a rigid bronchoscope and an argon plasma coagulator on standby. Following laryngeal mask airway placement, covered self-expandable metallic stents (Ultraflex; Boston Scientific, Marlborough, MA, USA) were inserted using a guide wire. The diameter of the inserted stents ranged between 16 and $18 \mathrm{~mm}$ and their length ranged from 60 to $80 \mathrm{~mm}$ (Table II).

\section{Results}

Symptom relief. After stenting, immediate symptom relief and sufficient airway enlargement was achieved in group B within a few days; however, $\sim 3$ months were required in group $A$ (tracheal stenosis at the cervical level caused by surrounding thyroid cancer, Fig. 2). Despite vocal cord paralysis, improvement of PS was achieved in all cases after stent placement 
Table I. Clinical characteristics of the 5 patients.

\begin{tabular}{lc}
\hline Characteristics & Data \\
\hline Gender, no. & \\
Male/female & $4 / 1$ \\
Age, years \pm SD (range) & $66 \pm 16(50-82)$ \\
Histological type, no. & \\
Papillary carcinoma & 3 \\
Medullary carcinoma & 1 \\
Anaplastic carcinoma & 1 \\
Initial tumor/recurrence, no. & $2 / 3$ \\
Vocal cord paralysis, no. & \\
Absent/unilateral/bilateral & $1 / 2 / 2$ \\
\hline
\end{tabular}

SD, standard deviation.

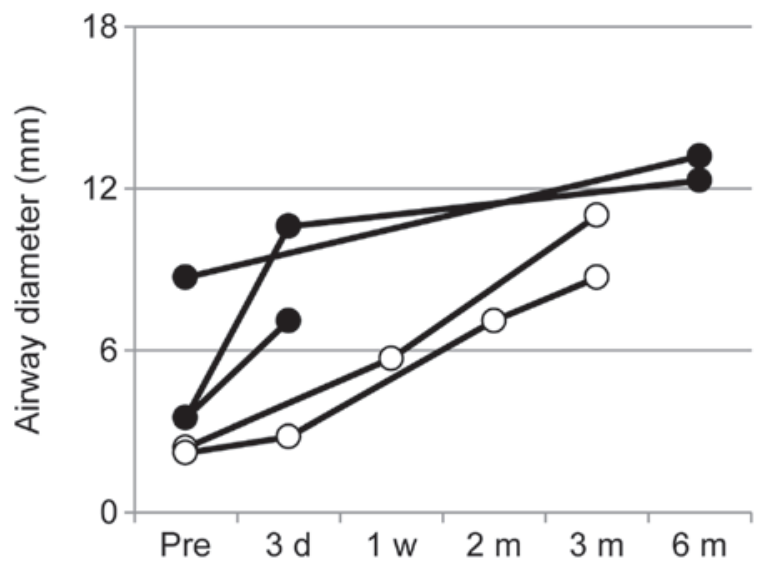

Figure 2. Enlargement of the airway after stenting. Open circles, changes in diameter of the airway in group A (patients 1 and 2); and closed circles, changes in diameter of the airway in group B (patients 3-5).

(Fig. 3). Three patients also exhibited an improvement of pulmonary function reflected in improved PEF, whereas one patient exhibited an improvement in $\% \mathrm{FEV}_{1.0}$.

Course after stent placement. During the follow-up period, the patient with anaplastic thyroid cancer (patient 4) succumbed due to rapid progression of the disease 2 months after stent placement. Including this case, 3 patients in total (patients 1 , 3 and 4) succumbed to the disease within 8 months, due to deterioration of their general condition caused by the carcinoma (papillary and medullary carcinoma). Patient 3 required tracheostomy 6 months after stenting due to tumor growth. We have been following up the remaining 2 patients with papillary carcinoma (cases 2 and 5) for 27 and 21 months, respectively, and the patients remain dyspnea-free (Table II). However, additional tracheostomy was required in patient 2 due to proximal granulation tissue formation and progressive bilateral vocal cord paralysis.

Granulation tissue formation. All the patients were administered tranilast to prevent granulation tissue formation after stenting. However, granulation tissue at both ends of the stent developed in 3 patients (Table III). Patient 2, who suffered from papillary carcinoma and poorly-controlled diabetes mellitus, experienced rapid and repeated granulation tissue formation. An additional metallic stent was inserted to cover the granulation tissue at the bare distal portion of the initial stent 9 months later and tracheotomy was performed immediately below the cricoid cartilage 1 month later, due to granulation tissue formation at the bare proximal portion and progressive bilateral vocal cord paralysis. In patient 5, granulation tissue developed at the bare proximal portion 6 months after stent placement (Fig. 4) and local treatment with prednisolone (PSL) and mitomycin C (MMC) was successful and resulted in regression.

Sputum retention. Retention of sputum and dried secretions inside the stent required intervention in patients 2 and 4 . As difficulties with sputum expectoration aggravated the dyspnea, emergency bronchoscopic lavage was performed in both cases. In patient 4 , in particular, the production of bloody sputum at the site of anaplastic carcinoma invasion could not be adequately controlled with the use of the covered metallic stent. Consequently, solidified adhesive secretion-coagulation deposits developed and obstructed the stent after 8 days. This coagulated adherent deposit was removed with a metal brush under general anesthesia.

Bacterial colonization inside the stent. Table III shows the results of the sputum culture. Although clarithromycin was administered to the patients to prevent infection immediately after stenting, all the stents were colonized by bacteria. Pseudomonas aeruginosa was detected in all patients, except patient 2. Methicillin-resistant Staphylococcus aureus (MRSA) was detected in patient 2 and Klebsiella oxytoca, Streptococcus pneumoniae and methicillin-sensitive Staphylococcus aureus were detected in patient 5. Levofloxacin hydrate was added to the treatment regimen for patient 5 but it was not effective in controlling colonization.

\section{Discussion}

The type of stent most suitable for relieving upper airway stenosis secondary to thyroid cancer remains controversial. Noppen et al (3) reported 30 patients with airway stenting for tracheal obstruction secondary to thyroid disease, consisting of 17 with benign disease and 13 with malignancies. The type of stent used in that study was mainly silicone stents, Dumon or Tygon-Noppen stents for the 13 cancer patients and an Ultraflex stent for replacement in 1 case where the Dumon stent had migrated. The Tygon-Noppen stent is a screw-thread silicone stent modified from an esophageal stent, designed not to migrate (4). Ultraflex stenting was also discussed in 3 patients with benign disease, due to 'specific stenosis characteristics (stenoses of variable axis diameter)'. Since thyroid cancer has a relatively indolent course, Tsutsui et al (5) hypothesized that the durable and replaceable nature of silicone stents may provide good results and compared 45 stents (12 silicone, 20 metallic and 13 T-tubes) in 35 patients with thyroid cancer. However, among 10 patients in whom the silicone stents had been placed in close proximity to the cricoid cartilage, 5 suffered from supraglottic stenosis and 4 from 
Table II. Type of airway stenosis and stent size.

\begin{tabular}{|c|c|c|c|c|c|c|}
\hline \multirow[b]{2}{*}{ Group } & \multirow[b]{2}{*}{ Patient } & \multirow{2}{*}{$\begin{array}{l}\text { Age (years), gender } \\
\text { histological type }\end{array}$} & \multicolumn{2}{|c|}{ Stenosis } & \multirow{2}{*}{$\begin{array}{l}\text { Vocal cord } \\
\text { paralysis }\end{array}$} & \multirow{2}{*}{$\begin{array}{l}\text { Stent size } \\
\quad(\mathrm{mm})\end{array}$} \\
\hline & & & Diameter, mm (\%) & Length, $\mathrm{cm}$ & & \\
\hline \multirow[t]{2}{*}{ A } & 1 & $\begin{array}{c}50, \mathrm{M} \\
\text { Medullary }\end{array}$ & $3(60)$ & 2 & Bilateral & $16 \times 60$ \\
\hline & 2 & $\begin{array}{c}70, \mathrm{~F} \\
\text { Papillary }\end{array}$ & $2(90)$ & 3 & Bilateral & $18 \times 60$ \\
\hline \multirow[t]{3}{*}{ B } & 3 & $\begin{array}{c}\text { 82, M } \\
\text { Papillary }\end{array}$ & $3(70)$ & 4 & Absent & $18 \times 60$ \\
\hline & 4 & $\begin{array}{c}50, \mathrm{M} \\
\text { Anaplastic }\end{array}$ & $3(70)$ & 7 & Right & $18 \times 80$ \\
\hline & 5 & $\begin{array}{c}\text { 76, M } \\
\text { Papillary }\end{array}$ & $3(80)$ & 2 & Left & $18 \times 60$ \\
\hline
\end{tabular}

Group A, patients with stenosis at the cervical level caused by thyroid cancer; group B, patients with stenosis at the mediastinum caused by thyroid cancer or metastatic lymph nodes.

Table III. Complications and prognosis.

\begin{tabular}{|c|c|c|c|c|}
\hline Patients & Granulation tissue & Retained secretions & Colonization & Prognosis \\
\hline 1 & Both ends (3 mo) & No & P. aeruginosa & $8 \mathrm{mo}, \mathrm{D}$ \\
\hline 2 & Both ends (9 mo) & Yes & $\begin{array}{l}\text { P. aeruginosa } \\
\text { MRSA }\end{array}$ & $\begin{array}{c}27 \text { mo, } \mathrm{A} \\
(10 \mathrm{mo}, \text { tracheostomy })\end{array}$ \\
\hline 3 & Absent & No & P. aeruginosa & $\begin{array}{c}8 \mathrm{mo}, \mathrm{D} \\
(6 \mathrm{mo}, \text { tracheostomy })\end{array}$ \\
\hline 4 & Absent & Yes & $\begin{array}{l}\text { P. aeruginosa } \\
\text { K. oxytoca }\end{array}$ & $2 \mathrm{mo}, \mathrm{D}$ \\
\hline 5 & Both ends (6 mo) & No & $\begin{array}{c}\text { MSSA } \\
\text { St. pneumoniae }\end{array}$ & $21 \mathrm{mo}, \mathrm{A}$ \\
\hline
\end{tabular}

Mo, months; P. aeruginosa, Pseudomonas aeruginosa; D, deceased; A, alive; MRSA, methicillin-resistant Staphylococcus aureus; K. oxytoca, Klebsiella oxytoca; MSSA, methicillin-sensitive Staphylococcus aureus; St. pneumoniae, Streptococcus pneumoniae.

A

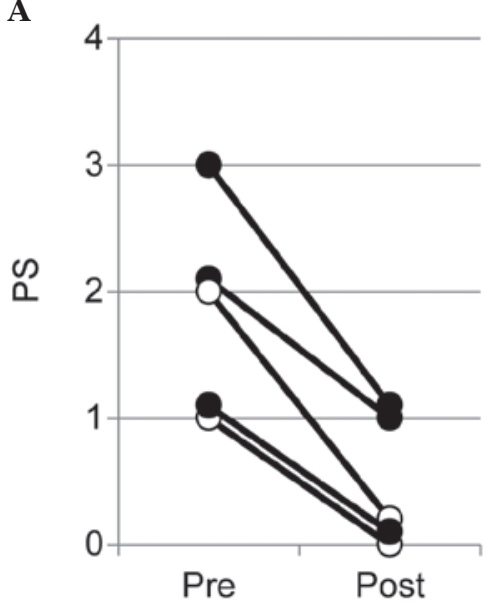

B

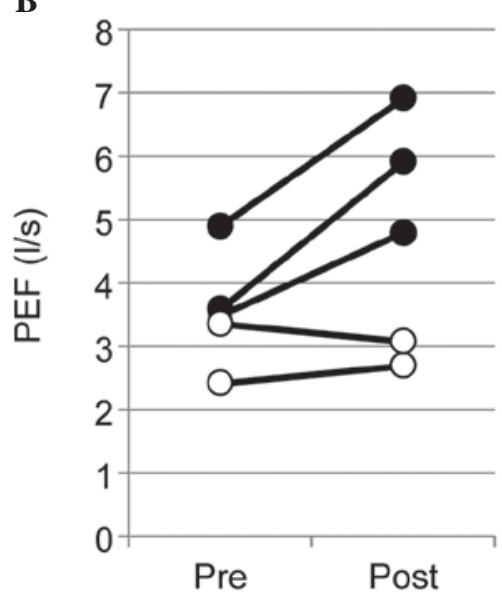

C

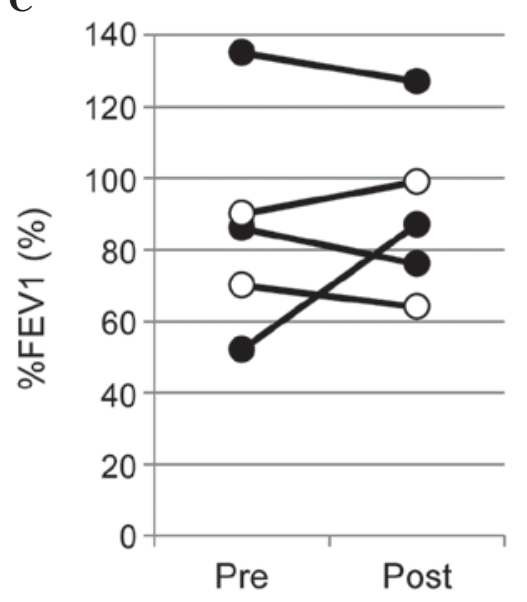

Figure 3. Changes in subjective and objective symptoms after stenting. (A) Improvement of performance status (PS) in all cases after stenting. (B) Group B (closed circles) exhibited improvements in peak expiratory flow (PEF), but this was not the case in group A (open circles), possibly due to bilateral vocal cord paralysis. (C) Improvement in percent predicted forced expiratory volume in $1.0 \mathrm{sec}\left(\% \mathrm{FEV}_{1.0}\right)$ in one case (patient 4$)$. 


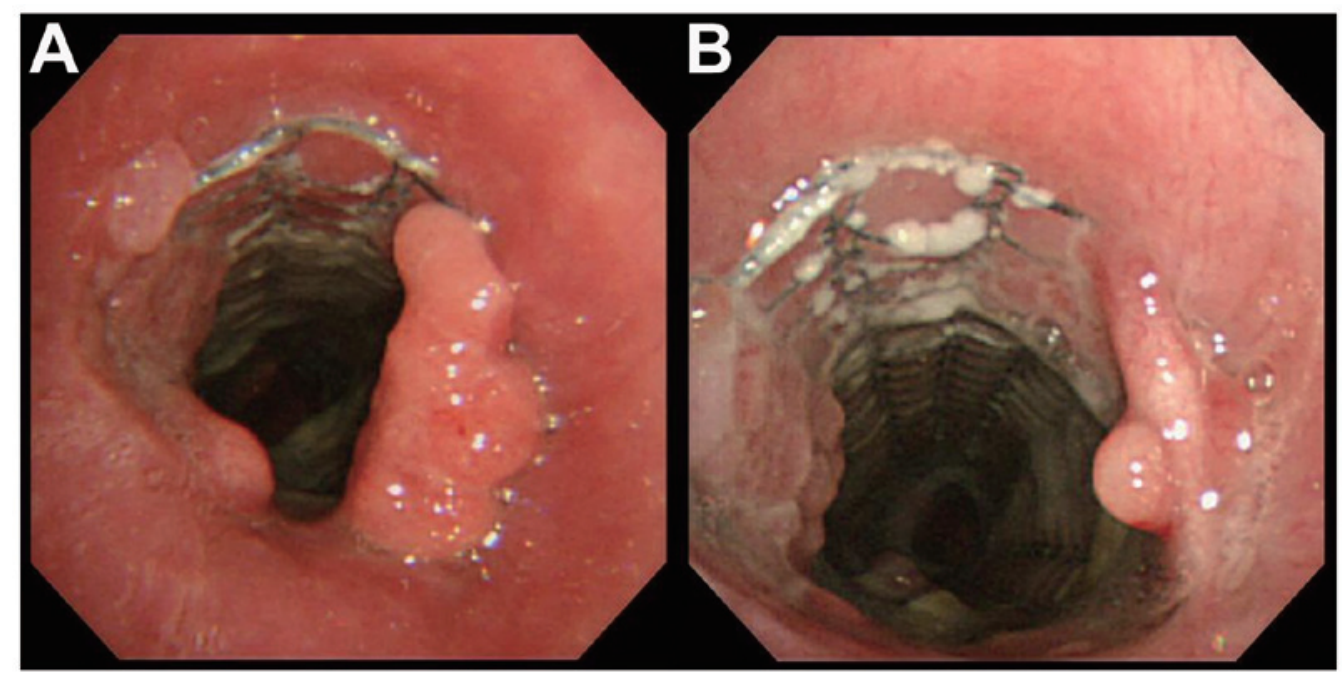

Figure 4. (A) Granulation tissue at the proximal end of the stent in patient 5, 11 months after stent placement. (B) Decreased granulation tissue, 3 months after topical use of mitomycin $\mathrm{C}$ and prednisolone.

stent migration. The authors concluded that use of the uncovered Ultraflex stent had fewer critical complications compared to the silicone stents. Kim et al (6) reported that the placement of covered retrievable self-expandable nitinol stents was safe and effective in patients with malignant $(n=7)$ or benign $(n=2)$ thyroid disease. Although one of their three types of stents was designed with barbs at the middle to prevent migration, the stent migrated in 2 of 4 such patients and stent replacement was required twice in each patient.

We selected covered Ultraflex self-expandable nitinol stents for all the cases, since we consider this type to be more appropriate compared to silicone stents in cases of extrinsic tracheal compression (7). These stents may also be more efficient in preventing tumor ingrowth through the mesh and are expected to be less harmful compared to other metallic stents with strong expansion. Therefore, potential rigid bronchoscope-related supraglottic stenosis in advanced thyroid cancer patients with edematous changes around their epiglottis may be avoided. However, in patients with extrinsic tracheal compression at the cervical level, surrounded by solid thyroid cancer tissue, a relatively long time was required to sufficiently enlarge the airway. Although the immediate enlargement benefits may be marginal with this approach, the patients benefitted psychologically from arresting the progress of dyspnea. Effective enlargement was achieved gradually over the next 3 months, without the requirement for intervention using a rigid bronchoscope.

Although the indications for stenting patients with anaplastic carcinoma may be debatable, one of our cases achieved a significant improvement of PS after stenting, despite the poor prognosis. Stent placement was useful not only for patients with well-differentiated thyroid cancer, but also for the acutely deteriorating anaplastic thyroid carcinoma patient. Thus, even only short-term placement of the stent may play an important role in palliative medicine.

Granulation tissue formation, sputum retention and bacterial colonization of the stents are the most common but burdensome complications of long-term stent placement $(5,8)$. It has been reported that airway colonization tends to be associated with sputum retention (3). Formation of granulation tissue was observed in 3 of our patients. Bacterial colonization was detected in all patients, 4 of whom had Pseudomonas aeruginosa. One patient had both Pseudomonas aeruginosa and MRSA and frequently suffered from dyspnea due to sputum retention within the stent. That patient required urgent bronchoscopic lavage. Granulation tissue was observed frequently at the proximal or the distal bare part of the otherwise covered stents. Saad et al (8) described 3 risk factors for developing granulation tissue with self-expandable metallic stents as follows: i) improperly sized stents (undersized, with excessive friction; or oversized, with excessive radial pressure); ii) exuberant bronchial mucosal inflammation; and iii) placement of any type of stent in the subglottic trachea. The mechanisms responsible for granulation tissue formation at the bare parts of the metallic stents have not been identified thus far; therefore, there are no known means of prevention. We hypothesized that the mechanisms may be similar to those occurring in chronic wounds (9). Prolonged periods of inflammation in wound healing are considered to significantly affect malgranulation. Factors which prolong inflammation include the following: i) general conditions due to metabolic disorders, nutritional problems and wasting syndromes; ii) hypoxia caused by respiratory or circulatory disorders; iii) interruption of the circulation due to ischemic disease, congestion, or lymphedema; iv) continuous infection; and v) secondary injury caused by continuous external pressure or friction (10). Although we considered the stents placed in all our patients to be appropriately sized, as documented by follow-up using 3DCT and/or bronchoscopy, granulation tissue developed in 3 patients. The pressure and friction exerted by these metallic stents and the persistent infection possibly participated in triggering the formation of granulation tissue. According to the abovementioned hypotheses, patients with thyroid cancer are considered to be at high risk of granulation tissue formation. The subglottic trachea is often involved in the stenotic lesion caused by thyroid cancer, exuberant bronchial mucosal inflammation or continuous infection occasionally accompanies these phenomena due to vocal cord paralysis and 
congestion or lymphedema may be associated with advanced thyroid cancer, which may lead to supraglottic stenosis after rigid-bronchoscopic procedures (5). Levofloxacin and tranilast had been administered to the patients reported in this study, in order to prevent granulation tissue formation $(11,12)$, but proved to be ineffective. In our patients, local injection of PSL and topical use of MMC (14) were successful in controlling granulation tissue. Therefore, testing this regimen in more patients may be of value.

Tsushima et al (14) reported a patient with metallic stents placed to relieve tracheobronchial stenosis secondary to thyroid cancer; the patient suffocated at home 56 days after stent placement, due to sputum retention associated with vocal cord paralysis. In our study, patient 2 frequently suffered from obstruction in the stents due to viscous secretions, which had to be repeatedly removed by bronchoscopy. In patient 4 , the anaplastic thyroid tumor was hemorrhagic and a large number of clots and viscous secretions obstructed the stent 8 days after placement. We removed the clots by bronchoscopy under general anesthesia. Noppen et al (15) recommended inhalation of aerosolized saline solution twice daily to avoid the accumulation of dried secretions inside the stents. All the patients in this study had been instructed to follow similar procedures; however, frequent observation of the stent interior remained indispensable. Dried secretions or sputum retention should be taken into consideration, particularly for patients with vocal cord paralysis or hemosputum.

This study demonstrated that the placement of self-expandable metallic stents to relieve central airway stenosis secondary to advanced thyroid cancer is a beneficial treatment, even in terminal-stage disease or in patients with anaplastic thyroid carcinoma. In conclusion, particularly with reference to differentiated thyroid tumors, as such patients have a relatively good prognosis in general, periodic and long-term bronchosopic follow-up is required to control for complications, including bacterial colonization, sputum retention and granulation tissue formation.

\section{Acknowledgements}

This study was supported in part by a Grant-in-aid for Scientific Research (C) no. 26462140 from the Japanese Ministry of Education, Culture, Sports, Science and Technology.

\section{References}

1. Japanese Society of Thyroid Surgery/Japanese Society of Endocrine Surgeons: Incidence, morbidity and mortality of thyroid carcinoma in Japan. In: Guidelines For Management of Thyroid Tumor. Japanese Society of Thyroid Surgery/Japanese Society of Endocrine Surgeons (eds). Kanehara Press, Tokyo, pp21-26, 2010 (In Japanese).

2. Noguchi S, Murakami N and Kawamoto H: Classification of papillary cancer of the thyroid based on prognosis. World J Surg 18: 552-557, 1994.

3. Noppen M, Pierard D, Meysman M, Claes I and Vincken W: Bacterial colonization of central airways after stenting. Am J Respir Crit Care Med 160: 672-677, 1999.

4. Noppen M, Meysman M, Claes I, D'Haese J and Vincken W: Screw-thread vs. Dumon endoprosthesis in the management of tracheal stenosis. Chest 115: 532-535, 1999.

5. Tsutsui H, Kubota M, Yamada M, et al: Airway stenting for the treatment of laryngotracheal stenosis secondary to thyroid cancer. Respirology 13: 632-638, 2008.

6. Kim WK, Shin JH, Kim JH, Song JW and Song HY: Management of tracheal obstruction caused by benign or malignant thyroid disease using covered retrievable self-expandable nitinol stents. Acta Radiol 51: 768-774, 2010.

7. Nomori H, Kobayashi R, Kodera K, Morinaga S and Ogawa K: Indications for an expandable metallic stent for tracheobronchial stenosis. Ann Thorac Surg 56: 1324-1328, 1993.

8. Saad CP, Murthy S, Krizmanich G and Mehta AC: Self-expandable metallic airway stents and flexible bronchoscopy: long-term outcomes analysis. Chest 124: 1993-1999, 2003.

9. Yuasa R, Takagi K, Takahashi S, et al: Treatment of granulation after airway stent placement for thyroid papillary carcinoma in a single case. J Jpn Soc Resp Endoscopy 33: 458-463, 2011 (In Japanese with English abstract).

10. Japanese Society of Pressure Ulcers: Overview of pressure Ulcers. In: Guideline for Prevention and Management of Pressure ulcers. Japanese Society of Pressure Ulcers (ed). Kanehara Press, Tokyo, pp18-23, 2009 (In Japanese).

11. Terada Y, Sakaguchi Y, Kono T, Nohara J and Noguchi T: Intrabronchial foreign body extracted with tranilast and corticosteroid. Ann Thorac Surg 86: 1686-1688, 2008.

12. Suzawa H, Kikuchi S, Arai N and Koda A: The mechanism involved in the inhibitory action of tranilast on collagen biosynthesis of keloid fibroblasts. Jpn J Pharmacol 60: 91-96, 1992.

13. Penafiel A, Lee P, Hsu A and Eng P: Topical mitomycin-C for obstructing endobronchial granuloma. Ann Thorac Surg 82: e22-e23, 2006

14. Tsushima K, Wakamatsu T, Furihata K, et al: A case of thyroid cancer treated with stent-in-stent placement to relieve tracheobronchial stenosis. J Jpn Soc Resp Endoscopy 24: 86-90, 2002 (In Japanese with English abstract).

15. Noppen M, Poppe K, D'Haese J, Meysman M, Velkeniers B and Vincken W: Interventional bronchoscopy for treatment of tracheal obstruction secondary to benign or malignant thyroid disease. Chest 125: 723-730, 2004. 